\title{
FASART: An iterative reconstruction algorithm with inter-iteration adaptive NAD filter
}

\author{
Ziying Zhou ${ }^{\mathrm{a}}$, Yugang Li ${ }^{\mathrm{a}}$, Fa Zhang ${ }^{\mathrm{b}}$, and Xiaohua Wan ${ }^{\mathrm{b}, *}$ \\ ${ }^{a}$ Beijing Lab of Intelligent Information Technology, School of Computer Science, Beijing Institute of \\ Technology, Beijing 100081, China \\ ${ }^{\mathrm{b}}$ Key Lab of Intelligent Information Processing, Institute of Computing Technology, Chinese Academy \\ of Sciences, Beijing, China
}

\begin{abstract}
Electron tomography (ET) is an essential imaging technique for studying structures of large biological specimens. These structures are reconstructed from a set of projections obtained at different sample orientations by tilting the specimen. However, most of existing reconstruction methods are not appropriate when the data are extremely noisy and incomplete. A new iterative method has been proposed: adaptive simultaneous algebraic reconstruction with inter-iteration adaptive non-linear anisotropic diffusion (NAD) filter (FASART). We also adopted an adaptive parameter and discussed the step for the filter in this reconstruction method. Experimental results show that FASART can restrain the noise generated in the process of iterative reconstruction and still preserve the more details of the structure edges.
\end{abstract}

Keywords: electron tomography (ET), 3D reconstruction, iterative method, non-linear anisotropic diffusion (NAD) filter

\section{Introduction}

Transmission electron microscopy (TEM) can achieve the visualization of structural information of biological specimen (macromolecular structures, or cellular structures, etc.) in the nanometer range. Electron tomography (ET) refers to the technique in which transmission electron microscopes are employed to collect projections of a biological object from multiple directions which are then used for the reconstruction of the object by means of reconstruction methods [1]. However, there are several obstacles to acquire high-quality reconstructions, including a missing wedge due to incomplete data at high tilt angles and extremely noisy data caused by low doses of electrons.

Various reconstruction methods have been proposed to solve these reconstruction problems, such as weighted back-projection (WBP) [2] and some iterative reconstruction methods [3-6]. We further explored an iterative method named adaptive simultaneous algebraic reconstruction (ASART) [4], and

\footnotetext{
*Address for correspondence: Xiaohua Wan, Key Lab of Intelligent Information Processing, Institute of Computing Technology, Chinese Academy of Sciences, Beijing, China. Tel.: +86-15101037202; E-mail: bjwxiaohua@ gmail.com.
} 
found that it could sharpen the edge and generate high-contrast reconstructed results, but the results are always noisy so that it is necessary to consider extra methods for noise reduction.

Some traditional filters, such as Gaussian and median filters are usually adopted to reduce noise in the process of post-reconstruction. These filters can remove the high-frequency noise, but induce a smooth edge $[7,8]$. A nonlinear anisotropic diffusion (NAD) filter is used to denoise ET images since it can prevent blurring the edges of the images and producing artifacts when smoothing the images $[1,7,9,10]$. However, it is not enough to restrain the noise of ET images in the post-reconstruction phase since the noise is growing more obvious during the process of the iterative reconstruction. Besides, we need to carefully consider how to adopt the filter in the process of iterative reconstructions since redundant filters always lead to a blurred biological structure in the filtered images.

In this paper, an iterative reconstruction method with inter-iteration adaptive NAD filter called FASART based on ASART is proposed. A three-dimensional (3D) NAD filter is embedded into the process of iterative reconstructions in order to restrain the noise and further improve the quality of the final reconstruction. The reconstruction method with inter-iteration filter is introduced in section 2. Experimental results are described in section 3. The conclusion and discussion part are presented in section 4.

\section{Method}

\subsection{General iterative reconstruction process}

3D reconstruction of ET requires a set of projections acquired at different orientations with a small tilt increments $\left(1^{\circ}\right.$ or $\left.2^{\circ}\right)$ by the using of transmission electron microscope as shown in Figure 1 . An original 3D volume denoted by $\boldsymbol{V}^{(0)}$ is usually computed by back-projection technique (BPT) or filter back-projection technique (FBP). The iterative process to obtain $\boldsymbol{V}^{(k)}$ can be expressed in the following equation:

$$
\boldsymbol{V}^{(i)}=\boldsymbol{V}^{(i-1)}-\frac{A_{i} \boldsymbol{V}^{(i-1)}-p_{i}}{A_{i} \cdot A_{i}} A_{i} \quad \text { where } p_{i}=\sum_{j=1}^{N} a_{i j} v_{j} \quad i=1,2, \ldots, M
$$

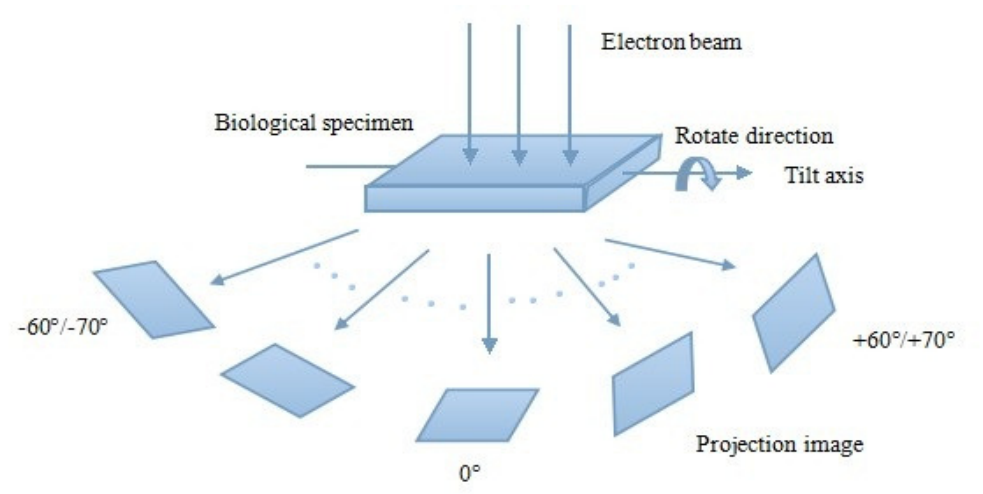

Fig. 1. Process of Projection. 
where $A_{i}=\left(a_{i 1}, a_{i 2}, \cdots, a_{i n}\right), v_{j}$ represents the $j_{t h}$ pixel in the original object. $a_{i j}$ represents the weighting factor and $p_{i}$ represents the $i_{t h}$ measured image of 3D volume. $\mathrm{M}$ and $\mathrm{N}$ represent the total number of rays and pixels respectively. If there is only one solution to the Eq. (1), the iteration will converge finally [11-13].

\subsection{Non-linear anisotropic diffusion filter}

Diffusion is a widely-used physical process in image processing. A partial diffusion equation (PDE) formulation was first proposed by [14], which is given below:

$$
\frac{\partial I}{\partial t}=\operatorname{div}(D \cdot \nabla I)
$$

where $\nabla I$ is the vector of gradient. The diffusivity $\mathrm{D}$ is defined as a function of struct tensor [15]:

$$
D=\left[\overrightarrow{v_{1}}, \overrightarrow{v_{2}}, \overrightarrow{v_{3}}\right] \cdot\left[\begin{array}{ccc}
\lambda_{1} & 0 & 0 \\
0 & \lambda_{2} & 0 \\
0 & 0 & \lambda_{3}
\end{array}\right] \cdot\left[\overrightarrow{v_{1}}, \overrightarrow{v_{2}}, \overrightarrow{v_{3}}\right]^{T} \quad \text { and } \quad J_{0}=\nabla I \cdot \nabla I^{T}
$$

The vectors $\overrightarrow{v_{1}}, \overrightarrow{v_{2}}$ and $\overrightarrow{v_{3}}$ represents the eigenvectors of $J_{0}$, where $\overrightarrow{v_{1}}$ and $\overrightarrow{v_{3}}$ corresponding to the maximum and minimum eigenvalue respectively. For a 3D ET image, $J_{0}$ is a $3 \times 3$ symmetric matrix which includes a vector $\overrightarrow{v_{1}}=\nabla I /|\nabla I|$ and corresponding eigenvalue $\mu_{1}=|\nabla I|^{2}$, and other zero eigenvalues. Sometimes, a smooth version of $J_{0}$ can also be used to improve the filtered image. $\lambda_{i}$ is a parameter closely related to the eigenvalues $\mu_{i}$. In general, the most commonly used diffusion approach is an edge enhancing diffusion (EED):

$$
\lambda_{1}=g(|\nabla I|), \lambda_{2}=g(|\nabla I|), \lambda_{3}=1 \quad \text { where } g(|\nabla I|)=\frac{1}{1+|\nabla I|^{2} / d^{2}}
$$

$J_{0}$ is used to compute the eigenvectors in EED. The main advantage of EED is edge detection and preservation.

\subsection{Iterative reconstruction with inter-iteration NAD filter}

Denoising filters have been employed in ET for many years. Some filters are added into the projection data and then these filtered projections are used to reconstruct 3D result [16]. Other filters are employed to process the reconstructed results to improve the quality of the final reconstructions [7,9]. Though the noise can be restrained in both methods, a filter used before the reconstruction process could eliminate some useful information and a filter used in the process of the post-reconstruction might lead to the over-smoothing problem.

In our work, we proposed a new iterative reconstruction algorithm with inter-iteration adaptive NAD filter called FASART based on adaptive simultaneous algebraic reconstruction method [4]. An adaptive NAD filter is adopted to restrain the noise of the reconstructed data in the iterative process. Then the denoised reconstructed result could be used in the next iteration. Although it costs extra time to process the iterative reconstructed result, the useful information in the initial tilt-series will not be removed by the filter and further FASART can suppress the over-smoothing result. 
To obtain a more exact model of the original object, we need to calculate the projection error between computed projection $P_{i}^{\prime}$ and real projection $P_{i}$, and then use this deviation to update the reconstructed result. It can be described as follows:

$$
V_{t}=V_{t}^{(0)}+\lambda \Delta P_{i} \quad \text { where } \Delta P_{i}=P_{i}-P_{i}^{\prime}
$$

where $\lambda$ represents the relaxation parameter and $V_{t}$ represents the reconstructed result in $t_{t h}$ iteration. A $3 \mathrm{D}$ NAD filter with an EED type is added into $V_{t}$ to obtain a more smooth reconstructed result $V_{t}^{\prime}$. The gradient threshold parameter d in EED is usually constant. However, the gradient value of the final image must have been changed greatly in the iterations. Hence, we propose an adaptive gradient threshold parameter $\mathrm{d}$ which is defined as follows:

$$
d=k \cdot s(|\nabla I|) \quad \text { where } s(|\nabla I|)=\sum_{i=1}^{n}\left|\nabla I_{i}\right|
$$

$s(|\nabla I|)$ represents the sum of the absolute gradient values on each Z-section of the reconstruction result and $\mathrm{k}$ is a threshold parameter. The details of the effect of different values of $\mathrm{k}$ and the number of the filters added into the iterative process will be discussed in section 3. With the adaptive scheme, each Z-section in a 3D volume has a different gradient threshold. If the reconstruction still does not achieve convergence after the using of filter, we will use $V_{t}^{\prime}$ as an initial volume and then repeat the filtered process until convergence. Although the filter can restrain the noise during the process of reconstructions, we need to carefully consider the number of the filters added in the process of iterative reconstruction because of the over-smoothing problem induced by the filters.

\section{Results}

To further study the property of FASART, we have done several experiments for different purpose. Here, two sets of microscopic images collected from FEI company's production-Tecnai 20 are used. The first is a mitochondria membrane structure and the second is caveolae of a procine aorta endothelial (PAE) cell. The size of two 3D volumes is $512 \times 512$ pixels and the number of the projection images are 59 and 222 , respectively.

\subsection{Adaptive parameters}

The gradient threshold parameter plays a major role in the diffusion process, since it are related with edge preserving and denoising. It is reasonable to choose a variable threshold compared with a constant threshold in each iteration since the gradient value for each reconstructed result for each iteration varies greatly. To study the influence of the gradient parameter on the reconstructed results, we have experimented with different values of $\mathrm{k}$ as described in Eq. (6).

From Figure 2(a), 2(c) and 2(d), we can see that there are some extreme points (e.g. white light spots) which can be regarded as artifacts marked in white square frame. In Figure 1(b), we can see that the image becomes more smooth with a $\mathrm{k}$ equal to 0.5 . This is because most of the pixels with noise have relatively higher gradient values. If we adopt $\mathrm{k}$ with a relative low value, the filter seems like an allpass filter and has few effect on the reconstructed results. But in the reconstructed results with ASART, 


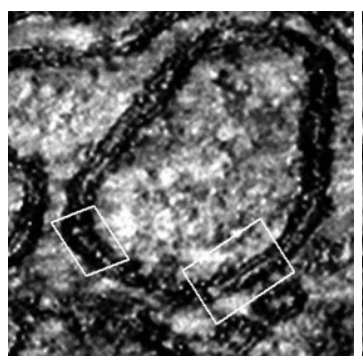

(a)

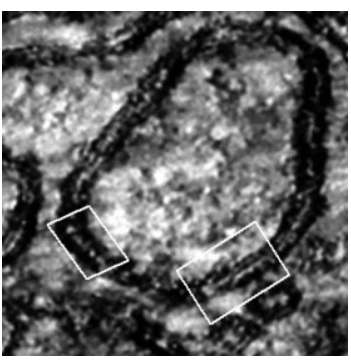

(b)

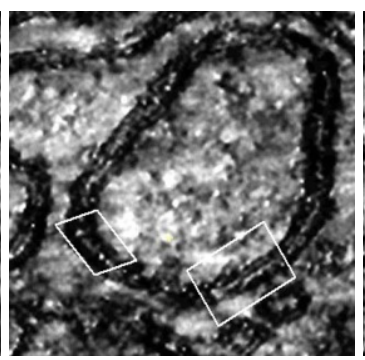

(c)

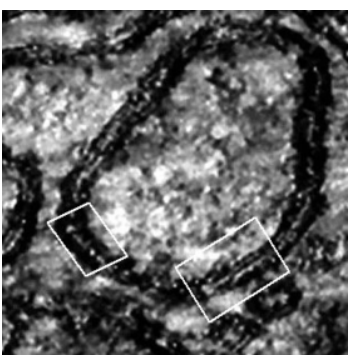

(d)

Fig. 2. Image processed with different gradient threshold values. (a) $d$ is a constant 1 (b) $k=0.5$ (c) $k=0.75$ (d) $k=2$.

there are always some extreme points with high values. With a much higher value of $\mathrm{k}$ in NAD filter, these light spots are regarded as a part of edges which will be kept completely. Therefore, considering the reconstructed results with ASART always have a high-contrast edges, it's not suitable to choose a relatively higher value for the gradient threshold. We need to consider a balance between preserving edges and restraining noise.

To obtain a high-quality images, we need to carefully consider how to add the filter into the process of iterative reconstructions, such as the number and step for adding the filters. We adopted a projection error criterion [3,4] to evaluate the quality of reconstructed results. It is often used as a standard to quantify the quality of reconstruction results, and a lower projection error value indicates a higher image quality. The projection error $\varepsilon$ is defined as follows:

$$
\varepsilon=\left\{\frac{1}{n} \sum_{i=1}^{n} \frac{\left(p_{i}-p_{i}^{\prime}\right)^{2}}{w_{i}}\right\}^{1 / 2}
$$

where $w_{i}=\overrightarrow{v_{i}} \cdot \overrightarrow{v_{i}}, \overrightarrow{v_{i}}$ is a j-dimensional vector. We calculated the final projection error defined in Eq. (7) for each way, the projection errors for ASART and FASART with different steps for adding k are displayed in Figure 3(a). From the figure, we can find that the filter could slow the speed of the convergence comparing the projection errors calculate from ASART and FASART. The projection errors of the result with the filter added 3 times per 3 iterations become larger with the iterative number increases, which

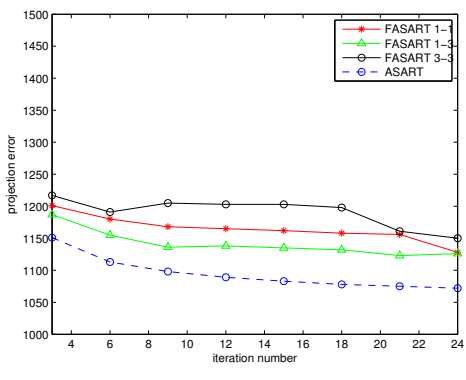

(a)

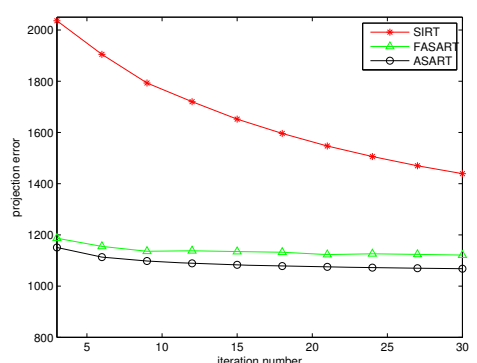

(b)

Fig. 3. Figure (a) is the projection error e of the results from FASART with: (1) the filter added once per 1 iteration; (2) once per 3 iterations; (3) 3 times per 3 iterations; (4) from ASART without filter. Figure (b) is the projection error e of the results from SIRT, FASART and ASART. 
means the number of the filter added can affect the convergence of iterations. The errors of the results with the filter added once per 3 iterations are smaller than those with the filter added once per 1 iteration. It seems to indicate that the filter distributed evenly can benefit for the quality of the results.

\subsection{Comparison and evaluation}

In Figure 4, the reconstructed results obtained from ASART, FASART, ASART with a post-processing NAD filter are shown respectively. Comparing the images, we can clearly see that the results with ASART have much noise and light spots. FASART can eliminate the light spots and further restrain the noise. The results with ASART and post-processing NAD filter in Figure 4(c) is more blurred than those with FASART in Figure 4(b). We could not see the details marked in white square rectangle clearly in Figure 4(c). Thus, with the same number of the NAD filters, the results reconstructed with FASART are more clear than those with ASART using a post-processing NAD filter.

To evaluate the quality of reconstructed results, we calculated the projection errors $\varepsilon$ of three methods's reconstructed results: ASART, a most popular iterative reconstruction method SIRT [3] and FASART, the results are shown in Figure 3(b). We can find that $\varepsilon$ of the reconstructions with FASART are a bit larger than those with ASART, but still are much smaller than those with SIRT. It shows that FASART can be convergent with an appropriate number and step of the filter added and achieve an improved visualization.

\section{Conclusion and discussion}

In this paper, we propose an iterative reconstruction method with inter-iteration adaptive NAD filter called FASART. We take advantage of an iterative reconstruction method ASART and a nonlinear anisotropic diffusion to resolve the blurring problem and improve the reconstructed results. FASART can

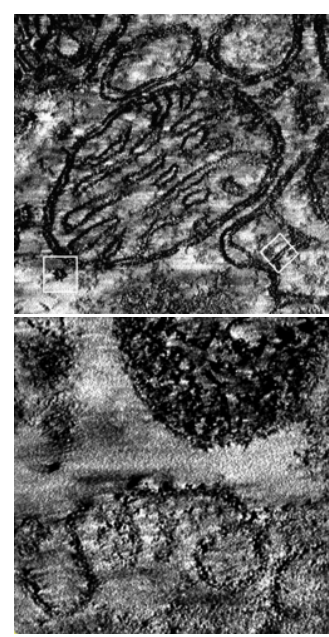

(a)

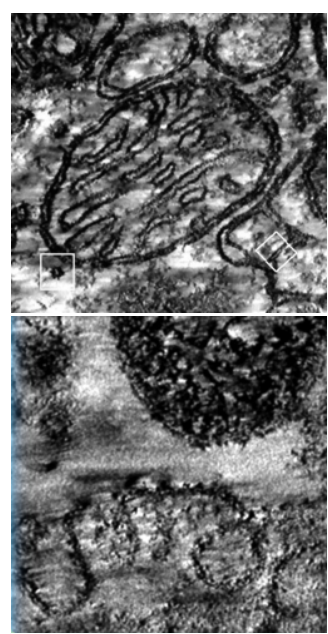

(b)

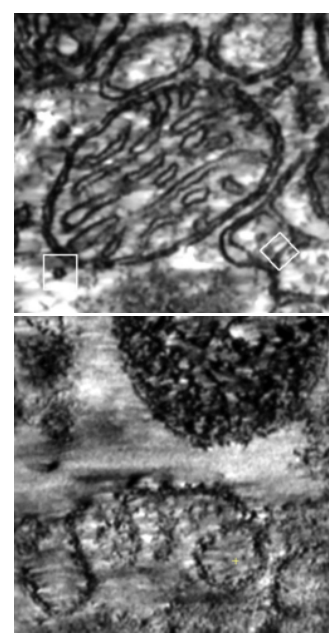

(c)

Fig. 4. The figures (a) shows the reconstructed results from ASART with 30 times iteration, figures (b) shows the results from FASART with one times filter per 3 times iteration, and figures (c) shows the results from post-processing reconstruction with 10 times NAD filter. 
reduce noise during the process of iterative reconstructions. Thus, more details of the structures can be well kept after the iterations.

Furthermore, there are several limits of FASART. First, NAD filter might eliminate some useful information of 3D volume. Therefore, we need to carefully choose the number of the filters added to promise FASART's convergence. It is important to consider the step of the filters embedded into the process of the iterative reconstructions because the scattered distribution of the filters can restrain the noise and keep the useful details. Second, the convergence speed of FASART is slower than that of ASART since it takes extra time to add the filter into the iterative reconstruction. Thus we are going to use parallelization of the filter to reduce the reconstruction time.

NAD filters which added into the process of iterative reconstructions can reduce the missing wedge resulted from an incomplete projection data. In our future work, we will consider other types of filters, such as Wiener filter and bilateral filter which have been widely used in ET.

\section{Acknowledgement}

This work is supported by the Specialized Fund for Joint Building Project of Beijing Municipal Education Commission (Grants No. 61070129), grants National Natural Science Foundation for China (Grants No. 61202210, 61232001, 61472397) and the Strategic Priority Research Program of the Chinese Academy of Sciences (Grant No. XDB08030202).

\section{References}

[1] J. Frank, Electron Tomography, Plenum, New York, 1992.

[2] M. Radermacher, Weighted back-projection methods, In J. Frank (Ed.), Electron tomography, Plenum, New York, pp. 91-115.

[3] P. Gilbert, Iterative methods for the three-dimensional reconstruction of an object from projections, Journal of theoretical biology 36 (1972), 105-117.

[4] X. Wan, F. Zhang, Q. Chu, K. Zhang, F Sun, Three-dimensional reconstruction using an adaptive simultaneous algebraic reconstruction technique in electron tomography, Journal of structural biology 175 (2011), 277-287.

[5] R. Marabini, G.T. Herman, J.M. Carazo, 3D reconstruction in electron microscopy using ART with smooth spherically symmetric volume elements (blobs), Ultramicroscopy 72 (1998), 53-65.

[6] A.H. Andersen and A.C. KakAndersen, Simultaneous algebraic reconstruction technique (SART): a superior implementation of the ART algorithm, Ultrasonic imaging 6 (1984), 81-94.

[7] A.S. Frangakis and R. Hegerl, Noise reduction in electron tomographic reconstructions using nonlinear anisotropic diffusion, Journal of structural biology 135 (2001), 239-250.

[8] A. Stoschek and R. Hegerl, Denoising of electron tomographic reconstructions using multiscale transformations, Journal of structural biology 120 (1997), 257-265.

[9] J.J. Fernandez and S. Li. An improved algorithm for anisotropic nonlinear diffusion for denoising cryo-tomograms, Journal of structural biology 144 (2003), 152-161.

[10] M.J. Black, G. Sapiro, D.H. Marimont, D. Heeger, Robust anisotropic diffusion, Image Processing, IEEE Transactions on 7 (1998), 421-432.

[11] R.S. Ramakrishnam, S.K. Mullick, R.K.S. Rathore, R. Subramanian, Orthogonalization, Bernstein polynomials, and image restoration, Applied optics 18 (1979), 464-468.

[12] G.N. Hounsfield, Method of an apparatus for examining a body by radiation such as $\mathrm{x}$ or gamma radiation, (1975).

[13] M. Slaney, A.C. Kak, L.E. Larsen, Limitations of imaging with first-order diffraction tomography, Microwave Theory and Techniques, IEEE Transactions on 32 (1984), 860-874.

[14] P. Perona, J. MalikPerona, Scale space and edge detection using anisotropic diffusion, IEEE Transactions on Pattern Analysis and Machine Intelligence 12 (1990), 629-639.

[15] J. Weickert, Scale-space properties of nonlinear diffusion filtering with a diffusion tensor, Report No. 110, Laboratory of Technomathematics, University of Kaiserslautern, P.O. Box 3049, 67653 Kaiserslautern, Germany, 1994.

[16] M. Maiorca, C. Millet, E. Hanssen, B. Abbey, Local regularization of tilt projections reduces artifacts in electron tomography, Journal of structural biology 186 (2014), 28-37. 